\title{
Racial Differences in DSM Diagnosis Using a Semi-Structured Instrument: The Importance of Clinical Judgment in the Diagnosis of African Americans
}

\author{
HAROLD W. NEIGHBORS \\ STEVEN J. TRIERWEILER \\ BRIGGETT C. FORD \\ JORDANA R. MUROFF
}

The University of Michigan

Journal of Health and Social Behavior 2003, Vol 43 (September): 237-256

Schizophrenia is diagnosed more frequently among African Americans while mood disorders are identified more often among whites. Such findings have raised serious questions about the accuracy of clinical judgment. This article analyzes data on 665 African American and white psychiatric inpatients using a semi-structured diagnostic instrument. The paper explores the relationship of patient race to schizophrenia, schizoaffective disorder, major depression, and bipolar disorder. The paper also explores the extent to which patient race is related to the manner in which clinicians link individual symptoms to diagnoses. Results indicate some significant race differences in diagnosis remain even when a semi-structured instrument and DSM criteria are used. whites, were more likely than African Americans to receive a diagnosis of bipolar disorder and less likely to be diagnosed with schizophrenia. There were no race differences in major depression. Some patterns of symptom attribution differed by race. The results are consistent with previous sociological research showing that patient race is related to diagnosis even when standardized diagnostic criteria are used. These findings underscore the importance of clinical judgment within the context of cross-race and cross-ethnic diagnosis. Clinical training programs must reduce ethnocentric bias by teaching the appropriate use of the sociocultural information necessary to employ DSM-IV's Cultural Formulation.

Sociologists have had a long-standing interest in racial differences in diagnostic categories (Loring and Powell 1988). ${ }^{1}$ Studies showing that schizophrenia more often is diagnosed among African Americans and that mood disorders more often are diagnosed among whites have raised serious questions about the accuracy of clinical judgment across race (Lawson, 1986; Neighbors, et al. 1989; Simon et al. 1973; Strakowski et al. 1996; Worthington, 1992). Studies of clinicians

* Address all correspondence to: Harold W. Neighbors, The School of Public Health, Department of Health Behavior and Health Education, M-5033 Thomas Francis Building SPH-II, The University of Michigan, 1420 Washington Heights, Ann Arbor, Michigan 48109-2029 using semi-structured instruments along with the careful application of criteria published in the most recent Diagnostic and Statistical Manual (DSM) of the American Psychiatric Association (American Psychiatric Association) typically find no race differences in diagnosis. Taken together, these findings have been interpreted as evidence of misdiagnosis, a situation that can have deleterious impact on treatment and outcome for patients. Although misdiagnosis is certainly a plausible explanation for such results, it does not clarify why clinicians implementing the same set of diagnostic criteria on the same patients often come to divergent diagnostic conclusions. At present, the field suffers from a tendency to stop short of probing for a deeper understanding of what 
factors contribute to racial differences in diagnosis.

The highly descriptive structure of the DSM-starting with the DSM-III (American Psychiatric Association, 1980)-provided researchers with an opportunity for a more rigorous examination of the sources of racial variation in the identification of mental disorders (Adebimpe 1981). DSM-III elaborated symptom criteria for particular disorders more carefully than earlier versions and, as a result, the mechanism for arriving at a particular diagnosis became a systematic inquiry into the presence or absence of symptoms comprising DSM criteria sets. The promise of DSM-III for addressing racial differences lay in its presumed equal applicability to all racial groups. From this standpoint, making modifications to the diagnostic criteria of DSM-III on the basis of patient race meant opening the door to stereotypes, bias, and ultimately misdiagnosis. DSM-III's attempt to limit clinical discretion was seen as a positive step because the diagnostic habits of clinicians working with African Americans had been suspect for quite some time (Fischer 1969; Garb 1997; Griffith 1996; Jones and Gray 1986; Kramer, Rosen, and Willis 1973; Raskin, Crook, and Herman 1975; Simon et al. 1973; Strakowski et al. 1996; Thomas and Sillen 1972; Welner et al. 1972).

Although DSM is an improvement over a completely idiosyncratic diagnostic process, it should not be applied mechanically in the clinical setting (American Psychiatric Association 1994; Kleinman 1996). This fact is especially important when confronting issues of crosscultural diagnosis. If cultural variation is to be taken seriously, clinicians must address the challenge of how to employ the cultural information (e.g., local idioms of distress, ethnic identity, degree of acculturation, religiosity, etc.) necessary to make an accurate diagnosis. DSM-III and DSM-III-R (American Psychiatric Association, 1980; American Psychiatric Association, 1987) were roundly criticized for assuming too much homogeneity and uniformity of symptom expression in patients from different racial or ethnic groups (Adebimpe 1981; Blue and Gaines 1992; Gaines 1992; Kirk and Kutchins 1992; Lock 1987). Despite improvements, DSM-IV (Ameican Psychiatric Association, 1994) is also criticized from a cultural standpoint (Mezzich et al. 1996; Tseng 1996). Only greater specificity regarding the development of a reasonable strategy for implementing DSM criteria across racial and ethnic groups will address these criticisms (Fabrega 1996; Kleinman 1996; Lukoff, Lu, and Turner 1992).

The purpose of this paper is to highlight the importance of clinical judgment by analyzing diagnostic data derived from a semi-structured instrument, the DSM-III-R Symptom Checklist (Hudziak et al. 1993). The paper provides a detailed exploration of the relationship of patient race to the diagnoses of schizophrenia, schizoaffective disorder, major depression, and bipolar disorder in a sample of African American and white psychiatric inpatients. The analysis goes beyond the typical crossclassification of race with disorder to probe the extent to which patient race is also related to the manner in which clinicians link individual symptoms (comprising the various DSM criteria sets) to a particular diagnostic category. In so doing we raise important questions concerning the appropriate manner in which DSM criteria are applied in the context of race.

\section{BACKGROUND}

The notion that African Americans are at higher risk for misdiagnosis than whites gained prominence with the publication of a series of papers in the early 1980s (Adebimpe 1981; Adebimpe 1982; Adebimpe, Klein, and Fried 1981; Bell and Mehta 1980; Bell and Mehta 1981). The concern about misdiagnosis emanates from two primary sources. First, the concern is grounded in a particular line of reasoning in interpreting existing diagnostic evidence. Many studies that look at diagnosis within a hospital setting have found that schizophrenia is diagnosed more frequently among African Americans and that mood disorders are diagnosed more often among whites (Lipton and Simon 1985; Liss et al. 1973; Mukherjee et al. 1983; Neighbors et al. 1999; Raskin, Crook and Herman 1975; Simon et al. 1973; Snowden and Cheung 1990; Strakowski et al. 1996; Tonks, Paykel and Klerman 1970; Welner, Liss and Robins 1973; Welner et al. 1972). The term "over-diagnosis" has been used to refer to the findings related to schizophrenia while "under-diagnosis" has been used to describe the findings on mood disorders. In turn, the conclusion of misdiagnosis and clinician bias has been based on these same bivari- 
ate relationships. The widespread interpretation that these findings are problematic reflects an implicit assumption of equal prevalence across race of all disorders in any given treatment setting, an assumption that is questionable given the evidence of a lower community prevalence of mood disorder for African Americans and the fact that, among those with mood disorders, African Americans are less likely than whites to seek mental health treatment (Sussman, Robins, and Earls 1987; U.S. Department of Health and Human Services 2001).

The second source of concern about misdiagnosis are studies of diagnostic disagreement across differing types of diagnostic interviews. Such studies show that, while African Americans are more likely than whites to receive a hospital diagnosis of schizophrenia, clinicians using semi-structured instruments and DSM criteria typically find no race differences in diagnosis when re-evaluating the same group of patients (Neighbors et al. 1999; Strakowski, Shelton, and Kolbrener 1993). Although many have attributed these findings to misdiagnosis, the evidence is far from conclusive; it may be premature to interpret these data as definitive evidence of misdiagnosis (Adebimpe 1981). Such an interpretation depends on accepting the assumption that clinicians using semi-structured diagnostic instruments, which presumably influence them to pay closer attention to DSM criteria, are more accurate than the typical diagnostic procedures employed in clinical settings. The very use of the term misdiagnosis implies a clear idea of which diagnosis is correct and which one is incorrect. Unfortunately, this assumptions is unfounded; in the absence of a gold standard, if two reasonable diagnostic processes disagree, it may not be clear that one procedure is superior (more accurate or valid) to the other.

Although the failure of the research psychiatrists to detect significant group differences in psychopathology casts some doubt on the hospital diagnoses (in this study) ... the alleged superiority of one diagnostic method over another is always open to debate. However, a structured mental status examination is much more likely to extract complete information, presumably with a consequent increase in diagnostic accuracy. (Adebimpe, Klein, and Fried 1981: 280)

The critical point is that comparing a presumably more structured and thorough procedure to the less specified (though not necessarily less accurate) sources of naturally occurring clinical impressions is not a definitive method for determining the degree to which African American patients are the victims of diagnostic error. The assumption of equal treated prevalence of all diagnostic categories by race implies that any identified race differences must be attributed to "misdiagnosis." From this line of reasoning, it is easy to see how one might conclude that research diagnoses produced by semi-structured instruments are the "correct" diagnoses. However, perhaps it is more appropriate to view them as "better" diagnoses. We know little about these research diagnoses except that they differ qualitatively from agency-based diagnoses in the process by which they are constructed. Nevertheless, the field has granted these outcomes superior status and, as a result, there is much we can learn from the instances of diagnostic divergence (agreement and disagreement across assessment procedures and patient race) they produce. Pinpointing the locations of the diagnostic divergence should suggest more precisely where race poses a particularly difficult diagnostic challenge for clinicians. Once identified, we can begin to examine these situations in detail, probing beyond diagnostic categories to understand how clinicians identify, interpret, and combine symptoms in the construction of a diagnostic hypothesis. In this way we will begin to identify the reasons that clinicians implementing a similar set of criteria sometimes come to divergent diagnostic conclusions.

Because psychiatric diagnosis relies so heavily on patient self-report, mental health clinicians must operate with some degree of clinical uncertainty (Fauman 1994). Clinical uncertainty opens the door to the possible influence of stereotypes that clinicians link to observable patient characteristics such as race (Smedley, Stith, and Nelson 2002). The social categorization process upon which stereotyping is based is essential because it allows people to handle the complexity and ambiguity that often characterize the cross-racial clinical encounter. Unfortunately, this cognitive process is often inappropriate because most stereotypes associated with race are negative and therefore offensive. More importantly, it is difficult if not impossible to see how racial stereotyping could ever be beneficial to the clinical process. No doubt some clinicians possess negative attitudes and opinions about 
African Americans and it is very possible that such negative attitudes are responsible for over-pathologizing African American patients (van Ryn and Burke 2000). However, the research in this area is inconsistent and, as a result, it is not clear how such attitudes influence the psychiatric diagnostic process (Abreu 1999). It is clear, however, that due to the limited nature of the research on clinician bias, this topic is in dire need of additional study (U.S. Department of Health and Human Services 2001).

Another premise of the misdiagnosis hypothesis is that clinicians are not sensitive to racial and ethnic differences in symptom expression. Unfamiliarity with the cultural norms of African American behavior leaves clinicians vulnerable to their own personal biases (Jones and Gray 1986; Lawson 1986). Many feel that these biases are unintentional, stemming from limited academic and professional training (U.S. Department of Health and Human Services 2001). This training deficiency, in turn, results in an inability to modify clinical processes once cultural factors are found to be important (Carter 1995; Carter 1974; Sue 1998; Sue and Zane 1987). Race is felt to somehow blur the boundaries between mental disorders. That is, being African American may obscure the prototypical picture of disorder, placing practitioners in unfamiliar clinical territory. Thus, it becomes more difficult for clinicians to make the appropriate clinical distinctions (Chen, Swann, and Johnson 1998).

In previous research, we examined the relationship between race and the diagnosis of schizophrenia in psychiatric inpatients by comparing the diagnoses of trained research clinicians using the semi-structured DSM-III-R Checklist to diagnoses generated by standard hospital procedures. Agreement between these different diagnostic sources was low (Neighbors et al. 1999). Forty-four percent of the patients who were diagnosed as having schizophrenia by the hospital received a different diagnosis from the research clinicians. This pattern of re-diagnosis occurred for both African American and white patients. The kappa depicting diagnostic agreement (schizophrenia, not-schizophrenia) between the hospital and research diagnoses was .39. Among African Americans, agreement between the hospital diagnosis and the research diagnosis was kappa of .39. The kappa for white patients was .37 .

Despite the low level of agreement in this study, and contrary to other studies using structured diagnostic interviews, we found that patient race was related significantly to both the hospital's diagnoses and the research diagnoses (Neighbors et al. 1999). The research diagnosticians yielded a higher rate of schizophrenia for African Americans (39\% compared to $31 \%$ ), while whites were significantly more likely to receive a research diagnosis of mood disorder (33\% compared to $21 \%$ ). Such findings are provocative because they contradict the prevailing position that the use of a semistructured instrument along with DSM criteria eliminates the typical relationship of race to psychosis and mood. However, our previously reported results are consistent with sociological research showing that patient race is related to diagnosis even when "clear-cut" diagnostic criteria are used (Loring and Powell 1988).

In summary, the present paper provides a detailed exploration of the relationship of patient race to the diagnoses of schizophrenia, schizoaffective disorder, major depression, and bipolar disorder as produced by clinicians using a semi-structured instrument and DSMIII-R criteria. In order to explore possible causes of race differences in diagnostic outcomes, this paper also analyzes symptom-level predictors of diagnosis. We push beyond the crossclassification of race with disorder to probe the extent to which patient race is related to the manner in which clinicians make differential symptom attributions and link them to a particular diagnostic category. Previous work suggests that when using a very flexible diagnostic interview, clinicians apply different decision rules to African American patients in judging the presence of schizophrenia (Trierweiler et al. 2000). The present study addresses whether similar differences can be discerned from the semi-structured interview format.

\section{METHOD}

\section{Data}

Setting. This research was conducted at a 148 bed state psychiatric facility located in Detroit, Michigan. The hospital was a state accredited facility with an internationally rec- 
ognized training faculty for the full-spectrum of mental health personnel, including psychiatrists, psychiatric social workers, clinical psychologists, occupational therapists, nurses, and special education teachers. Because of its location in an urban setting, the hospital occupied a unique position as a major provider in a full range of socio-psychiatric services. The hospital's clinical facility was a natural laboratory setting for research to study the urban chronically mentally ill and, as such, provided a patient population comparable to that treated by other public urban hospitals.

Patients. African American and white adult inpatients with an admitting diagnoses of schizophrenia (including schizoaffective disorder but not schizophreniform) or mood disorder (including bipolar, manic episode, and major depression) were eligible to participate in the study. Each patient was interviewed by clinicians blind to the hospital's admitting diagnosis. A total of 665 patients completed a DSM-III-R Symptom Checklist interview. The data collection was approved by the Institutional Review Board at both the University of Michigan and at the hospital. Patient participation was entirely voluntary. In order to encourage participation, patients were provided modest monetary compensation (five dollars) for each completed interview. Patients were allowed to terminate the interview at any time for any reason without losing financial compensation. This is a large data collection when compared to other diagnostic studies of African Americans.

Interviewer Selection and Training. Research interviewers were recruited from three local psychiatric residency programs. Residents who expressed an interest in the study were interviewed by a senior clinical psychologist who served as an on-site clinical coordinator for the study. Eight interviewers (3 African American and 5 non-African American, including two white, one from Latin America, one Southeast Asian, and one West African), who were third and fourth year psychiatric residents, were trained for this study. Applicants for interviewing positions were screened for their experience and ability to work with low-income African American patients. Interviewers were carefully trained on how to approach patients, introduce the study, obtain written consent, and administer the diagnostic instrument.

\section{Instrument and Diagnoses}

A shortened version of the DSM-III-R Symptom Checklist was used in this study (Hudziak et al. 1993). The DSM-III-R Symptom Checklist is a comprehensive, semistructured clinical instrument that does not permit a diagnostician to prematurely "skip out" of pursuing a particular diagnostic category. It requires clinicians to cover the entire group of symptoms associated with a particular diagnostic category, thereby guaranteeing that the DSM criteria set for a particular diagnosis is actually examined. While the DSMIII-R Symptom Checklist does not require diagnosticians to ask questions verbatim, it does instruct the clinician to cover the range of symptoms associated with a particular diagnostic category. Clinicians were able to probe freely until they were satisfied they had gained a thorough understanding of the symptom in question, which they then coded as present or absent. Instrument instructions led the clinician through DSM-III-R inclusion and exclusion rules to facilitate making a final diagnostic judgment. The analyses reported here refer to the research clinician's primary diagnosis as determined upon completing the DSM-III-R Symptom Checklist. The primary diagnosis was assigned after considering all of the symptoms and preliminary diagnoses reviewed during the session. A more detailed description of the methods and procedures employed in this study can be found elsewhere (Thompson et al. 1996).

Analysis Strategy. Multivariate analyses of race and diagnosis, adjusting for demographic covariates, were performed using multinomial logistic regression. This procedure is used when the dependent variable is polytomous and nominal. The independent variables may be either continuous or categorical. The outcome variable measured the following diagnostic categories: schizophrenia, bipolar, depression, schizoaffective, or other. Schizophrenia served as the reference category against which all other outcomes were compared.

Symptom Attributions. We use the term attribution as a more general cognitive concept to refer to the judgment process employed by the clinicians (Heider 1958). Here, the process of clinician attribution involves the act of ascribing a symptom to a patient. If important differences exist between patient races in the way 
clinicians arrive at a particular diagnosis, such differences should be revealed in the multivariate patterns of association existing between symptom designations and diagnostic decisions for each patient race (Trierweiler et al. 2000). To explore this conjecture, a series of binomial logistic regression analyses was conducted. Two binary dependent variables were created: schizophrenia (1) versus other $(0)$; and bipolar disorder (1) versus other (0). In separate analyses, each dependent variable was predicted by symptoms designated as present (1) or absent (0) by clinicians using the DSM-III-R Symptom Checklist. The DSM-III-R Symptom Checklist is comprised of distinct sections for symptoms describing major depression, psychosis, and manic episode. Each symptom criteria set was treated separately in the analysis of each diagnosis (i.e., schizophrenia and bipolar).

Procedure. The analysis steps were as follows. First, a backward stepwise, likelihoodratio procedure was used to eliminate redundancies and to reduce the number of variables in the analysis $(p>.10$, two-tailed, for removal). Second, all two-way interactions between the reduced set of symptom checklist variables and patient race were tested using a backward stepwise approach for the interactions with the $p$ for removal set at greater than .20 (Hosmer and Lemeshow 1989). All relevant main effects were present in the analysis of interaction effects. The presence of a significant interaction indicated that the odds of the diagnosis differed by patient race given the designation of the symptom(s) in the interaction. When there was evidence for one or more interactions, logistic regression models were calculated separately for each race so that similarities and differences in the pattern of symptom attributions could be examined. Patient sex and education (years completed) were hierarchically controlled in all analyses, save those used to refine estimates of regression weights, in which case education was excluded as described below.

\section{RESULTS}

Table 1 presents the socio-demographic distributions for the data collection. The sample was predominantly African American and male. Specifically, the clinical sample was 81percent African American and 65 percent
TABLE 1. Percentage Distribution of Demographic Characteristics for Study Participants

\begin{tabular}{llc}
\hline \hline & $\%$ & $(\mathrm{n})$ \\
\hline Race & & \\
$\quad$ African American & 81.3 & $(540)$ \\
$\quad$ European American & 18.7 & $(124)$ \\
Sex & & \\
$\quad$ Female & 35.3 & $(235)$ \\
$\quad$ Male & 64.7 & $(430)$ \\
Education & & \\
$\quad 0-11$ years & 46.1 & $(285)$ \\
$\quad 12$ years and above & 53.9 & $(333)$ \\
Age & & \\
$\quad 18$ to 35 years of age & 47.5 & $(312)$ \\
$\quad 36$ years and above & 52.5 & $(345)$ \\
& & Total $\mathrm{n}=665$ \\
\hline
\end{tabular}

male. The sample was more evenly split in terms of education with 54 percent of respondents with 12 or more years of education. Finally, 52.5 percent of the sample was 36 years old or older. The African American and white patients did not differ in terms of education and gender. African American patients were significantly more likely than whites to be age 35 or younger (Neighbors et al. 1999).

\section{Diagnostic Results}

Table 2 shows the bivariate relationship of race to the hospital's admitting diagnosis. There was a statistically significant difference between African Americans and whites in the distribution of the admitting diagnoses $\left(\mathrm{X}^{2}=\right.$ 15.28, df $=4, p<.01$ ). Specifically, African Americans displayed a higher percentage of schizophrenia (44\% compared to $32 \%$ ) and a lower rate of bipolar disorder (5.4\% compared to $14.3 \%$ ) than the white patients. There were no appreciable race differences in major depression or schizoaffective disorder.

Table 3 shows the bivariate relationship of race to the research clinician's primary diagnosis produced using the DSM-III-R Symptom Checklist interview. Similar to the results in Table 2, there was a statistically significant difference between African Americans and whites in the distribution of the DSM-III-R primary research diagnoses $\left(\mathrm{X}^{2}=19.72\right.$, $\mathrm{df}=4, p<$ .001). Specifically, African Americans displayed a higher percentage of schizophrenia (33\% compared to $24 \%$ ) and a lower rate of bipolar disorder $(6.5 \%$ compared to $18.5 \%)$ than the white patients. There were no race dif- 
TABLE 2. Percent Distribution of Hospital Admitting Diagnoses by Race

\begin{tabular}{lcccccc}
\hline \hline Race & Schizophrenia & Schizoaffective & Depression & Bipolar & Other & $(\mathrm{n})$ \\
\hline African-American & 42.4 & 13.2 & 25.9 & 5.2 & 13.2 & $(536)$ \\
European American & 30.6 & 16.1 & 24.2 & 13.7 & 15.3 & $(124)$ \\
\hline
\end{tabular}

Note: $p<.01$ for $\chi^{2}$ test of association, $\chi^{2}=15.12, \mathrm{df}=4$ )

TABLE 3. Percent Distribution of DSM-III-R Symptom Checklist Primary Diagnoses by Race

\begin{tabular}{lcccrcr}
\hline \hline Race & Schizophrenia & Schizoaffective & Depression & Bipolar & Other & $(\mathrm{n})$ \\
\hline African-American & 32.8 & 6.3 & 14.2 & 6.5 & 40.2 & $(537)$ \\
European American & 24.2 & 7.3 & 14.5 & 18.5 & 35.5 & $(124)$ \\
\hline
\end{tabular}

Note: $p<.001$ for $\chi^{2}$ test of association, $\chi^{2}=19.72, \mathrm{df}=4$ )

ferences in major depression or schizoaffective disorder. Both African Americans and whites had a substantial proportion of "other" diagnoses $(35 \%$ and $40 \%)$.

In order to examine further the underpinnings of these findings, a multinomial logistic regression analysis was conducted where age, education, gender, and marital status were entered as control variables, and schizophrenia was designated as the comparison category for diagnoses of bipolar disorder, depression, schizoaffective disorder, and other. The results of the multinomial regression analysis are presented in Table 4 . Note that the primary differences described in Table 3 were corroborated in the multinomial logistic regression analysis. Race was a major variable distinguishing bipolar disorder from schizophrenia. whites were four times more likely $(\mathrm{OR}=4.36)$ to receive a diagnosis of bipolar disorder than African Americans.

Age and education were found to distinguish schizophrenia from other disorders in several of the multinomial comparisons. In particular, older patients were somewhat more likely to receive a diagnosis of schizophrenia across all comparisons. Additionally, education and marital status were significantly associated with the diagnosis of schizophrenia in comparison to a diagnosis of bipolar disorder.

TABLE 4. Odds Ratios for DSM-III-R Symptom Checklist Diagnoses Adjusting for Age, Sex, Education, Marital Status, and Race (logistic regression with schizophrenia as referent)

\begin{tabular}{|c|c|c|c|c|c|}
\hline Research Diagnosis & $\mathrm{B}$ & S.E. & Odds Ratio & Significance & $95 \% \mathrm{CI}$ \\
\hline \multicolumn{6}{|l|}{ Bipolar } \\
\hline Age & -.031 & .018 & .970 & .097 & $.935-1.006$ \\
\hline Education & .213 & .109 & 1.24 & .051 & $.999-1.53$ \\
\hline Gender $^{\mathrm{a}}$ & .390 & .339 & 1.48 & .250 & $.760-2.87$ \\
\hline Race $^{b}$ & 1.47 & .367 & 4.36 & .000 & $2.12-8.94$ \\
\hline Marital Status ${ }^{\mathrm{c}}$ & 1.33 & .638 & 3.77 & .038 & $1.08-13.16$ \\
\hline \multicolumn{6}{|l|}{ Depressive } \\
\hline Age & -.031 & .015 & .969 & .045 & $.940-.999$ \\
\hline Education & .015 & .082 & 1.14 & .116 & $.969-1.33$ \\
\hline Gender & .311 & .277 & 1.37 & .261 & $.793-2.35$ \\
\hline Race & .387 & .350 & 1.47 & .269 & $.741-2.92$ \\
\hline Marital Status & -.407 & .329 & .666 & .217 & $.349-1.27$ \\
\hline \multicolumn{6}{|l|}{ Schizoaffective } \\
\hline Age & -.040 & .020 & .960 & .043 & $.924-.999$ \\
\hline Education & -.037 & .091 & .964 & .687 & $.807-1.15$ \\
\hline Gender & 1.12 & .361 & 3.07 & .002 & $1.51-6.22$ \\
\hline Race & .586 & .445 & 1.80 & .188 & $.751-4.30$ \\
\hline Marital Status & -.288 & .438 & .750 & .512 & $.318-1.77$ \\
\hline \multicolumn{6}{|l|}{ Other } \\
\hline Age & -.023 & .012 & .977 & .048 & $.955-1.00$ \\
\hline Education & -.005 & .053 & .995 & .929 & $.897-1.11$ \\
\hline Gender & .074 & .212 & 1.08 & .727 & $.711-1.63$ \\
\hline Race & .258 & .273 & 1.30 & .343 & $.759-2.21$ \\
\hline Marital Status & -.148 & .261 & .863 & .571 & $.517-1.44$ \\
\hline
\end{tabular}

aThe reference category is male for gender

${ }^{\mathrm{b}}$ The reference category is black for race

${ }^{\mathrm{c}}$ The reference category is married for marital status 
Highly educated and single individuals also were less likely to be diagnosed with schizophrenia and more likely to receive a diagnosis of bipolar disorder.

\section{Symptom Attribution Results}

An initial examination of the overall logistic regression models for each symptom set showed that the major depression symptoms did not significantly predict bipolar disorder and showed no significant interactions with race in predicting schizophrenia. Therefore, the major depression symptoms were excluded from further analysis.

Schizophrenia results. Results for the schizophrenia variable were significant for both psychotic and manic episode symptoms. The initial model for all patients on the 19 psychotic symptoms predicting schizophrenia was significant $\left(\mathrm{X}^{2}=196.358, \mathrm{df}=19, p<.001\right)$, correctly classifying 79 percent of the diagnoses. The reduced model showed that this effect was almost completely accounted for by a subset of nine symptoms: loose associations, catatonic behavior, inappropriate affect, hallucinations with voice, vague speech, odd beliefs, unusual perceptions, schizophrenic symptoms for more than 6 mos., and organic factor $\left(\mathrm{X}^{2}=199.820\right.$, $\mathrm{df}=9, p<.001)$. Analysis of race by symptom interactions showed that symptom attribution patterns differed in the diagnoses of African American and white patients; seven of the nine interactions tested were statistically significant, with only the interactions of race with inappropriate affect and odd beliefs failing tests of statistical significance.

Table 5 shows estimates of the logistic regression weights and corresponding odds ratios for each race group. Entries in Table 5 were obtained by running the reduced model separately for each race and looking separately at models that positively and negatively predicted the diagnosis. This approach reduced the distorting effects of redundancies and missing cells on the standard errors of the regression coefficients. In addition, initial analyses were conducted controlling for both patient sex and education. However, education showed collinearities and interactions with various symptom variables that elevated the standard errors of the regression weights. Analyses showed that excluding education reduced most standard errors to appropriate levels (i.e., < 1.0) (see Hosmer and Lemeshow 1989) without affecting the substance of the overall findings. Therefore, the results in Table 5 were calculated without the education control.

The top left quadrant of Table 5 shows the results for psychotic symptoms predicting the diagnosis of schizophrenia. The designations hallucinations and delusions, which are primary positive symptom categories for psychosis and which differed in attribution rates between races (hallucinations: African American $=.46$, white $=.33, \mathrm{X}^{2}=6.654, \mathrm{df}=1, p<.01$; delusions: African American $=.42$, white $=.33, \mathrm{X}^{2}$ $=6.654$, $\mathrm{df}=1, p=.06$ ), did not predict the schizophrenia diagnosis in the multivariate context. Rather, the major predictors of schizophrenia in this sample were more specific symptoms attributions. African American and white patients were similar in that loose associations, vague speech, and a judgment that schizophrenic symptoms had existed for more than six months positively predicted the schizophrenia diagnosis. A judgment that an organic factor initiated or maintained the observed disturbance negatively predicted schizophrenia for both race groups. There were no differences between races in the rates at which any of the aforementioned symptoms were attributed. However, schizophrenia was predicted by hallucinations with voice and inappropriate affect only for African American patients. Attributions of hallucinations with voice were made more often to African American patients (.23) than to white patients (.15) ( $\mathrm{X}^{2}=4.288$, $\mathrm{df}=1, p<.05)$. Inappropriate affect showed no race differences in attribution rate.

The initial model for all patients on the 13 manic episode symptoms predicting schizophrenia was significant $\left(X^{2}=92.488, d f=13\right.$, $p<.001)$ correctly classifying 77 percent of the diagnoses. This result was almost entirely due to a subset of five symptoms $\left(\mathrm{X}^{2}=88.515, \mathrm{df}=\right.$ $5, p<.001$; correctly classifying $77 \%$ of the diagnoses): flight of ideas, distractibility, delusions without mood symptoms, mood disorder superimposed on schizophrenia, and organic factor. Analysis of the race by symptom interactions for this set showed significant interactions for distractibility, mood disorder superimposed on schizophrenia, and organic factor.

The extent to which the set of manic episode symptoms co-occurred with schizophrenia is 
TABLE 5. Logistic regression analyses of symptoms predicting dichotomous schizophrenia and bipolar illness variables.

\begin{tabular}{|c|c|c|c|c|c|c|c|c|}
\hline \multirow[b]{3}{*}{ Symptoms } & \multicolumn{4}{|c|}{ Schizophrenia } & \multicolumn{4}{|c|}{ Bipolar Illness } \\
\hline & \multicolumn{2}{|c|}{ African American } & \multicolumn{2}{|c|}{$\begin{array}{c}\text { Non- } \\
\text { African American }\end{array}$} & \multicolumn{2}{|c|}{ African American } & \multicolumn{2}{|c|}{$\begin{array}{c}\text { Non- } \\
\text { African American }\end{array}$} \\
\hline & B & Odds Ratio & $\mathrm{B}$ & Odds Ratio & B & Odds Ratio & B & Odds Ratio \\
\hline
\end{tabular}

Delusions

Hallucinations

Loose Associations

Catatonic Behavior

Inappropriate Affect

Bizarre Delusions

Hallucinations with Voice

Decreased Functioning

Social Withdrawal

Impairment in Role Functions

Peculiar Behavior

Impairment In Hygiene

Blunted Affect

Vague Speech

Odd Beliefs

Unusual Perceptions

Lack of Energy

Schizo Symptoms $>6 \mathrm{Mos}$

Organic Factor
Psychotic Symptoms

\begin{tabular}{|c|c|c|c|c|c|c|c|}
\hline - & & - & & - & & - & \\
\hline - & & - & & - & & - & \\
\hline $.59 *$ & 1.81 & $1.21 \dagger$ & 3.38 & - & & - & \\
\hline - & & - & & - & & $1.31 \dagger$ & 3.69 \\
\hline $.92 * *$ & 2.52 & - & & - & & $-1.39 *$ & .25 \\
\hline - & & - & & - & & - & \\
\hline $.89 * *$ & 2.44 & - & & - & & - & \\
\hline - & & - & & - & & - & \\
\hline - & & - & & - & & - & \\
\hline - & & - & & $-1.09^{*}$ & .34 & - & \\
\hline - & & - & & - & & $-1.17 \dagger$ & .31 \\
\hline - & & - & & - & & - & \\
\hline 一 & & - & & $-3.38 * * *$ & .03 & - & \\
\hline $.71 *$ & 2.04 & $1.88 * *$ & 6.52 & - & & - & \\
\hline 一 & & - & & - & & - & \\
\hline- & & - & & - & & - & \\
\hline - & & - & & - & & - & \\
\hline $79 * * *$ & 6.00 & $2.91 * * *$ & 18.36 & - & & - & \\
\hline $10 * * *$ & .12 & $-2.90 * * *$ & .06 & - & & - & \\
\hline
\end{tabular}

Elevated Mood

Irritable (Only)

Grandiosity

Decreased Need of Sleep

Hypertalkative

Flight of Ideas

Distractibility

Psychomotor Agitation

Destructive Overindulging

Impairment of Functioning

Delusions w/o Mood

Symptoms

Superimposed Schizophrenia

Organic Factor

Manic Episode Symptoms

\begin{tabular}{|c|c|c|c|c|c|c|c|}
\hline- & & - & & - & & - & \\
\hline- & & - & & - & & - & \\
\hline- & & - & & - & & - & \\
\hline - & & - & & - & & - & \\
\hline - & & - & & $1.76^{* * *}$ & 5.79 & $1.76^{* * *}$ & 5.79 \\
\hline - & & - & & - & & - & \\
\hline - & & $-1.87 * *$ & .16 & - & & - & \\
\hline - & & - & & $1.35 * * *$ & 3.86 & $1.35 * * *$ & 3.86 \\
\hline - & & - & & - & & - & \\
\hline- & & - & & - & & - & \\
\hline $.80^{*}$ & 2.23 & - & & - & & - & \\
\hline $.78 * * *$ & 5.95 & $3.08 * * *$ & 21.79 & $-2.14 * * *$ & .12 & $-2.14 * * *$ & .12 \\
\hline $20 * * *$ & .30 & $-3.15 * *$ & .043 & $-1.62 * * *$ & .20 & $-1.62 * * *$ & .20 \\
\hline
\end{tabular}

$\dagger p<.10,{ }^{*} p<.05,{ }^{* *} p<.01, * * * p<.001$

The symbol "- indicates a non-significant likelihood ratio $\chi^{2}$ in the analysis including all variables in the section. Notes: Patient sex was controlled in all analyses. Table entries are for race comparisons only. Psychotic and manic episode symptoms were analyzed separately. Within those analyses, positively and negatively weighted variables, as indicated in the overall analyses, were run separately for each set of predictors.

There were no race by symptom interactions for the analysis of manic episode symptoms predicting bipolar illness diagnoses. Therefore, entries for both patient races are identical in the lower right quadrant of the table - both weights and significance tests are from the full sample model.

displayed in the lower left of Table 5. A judgment that an observed mood disturbance was superimposed on schizophrenia or schizophrenia related disorder positively predicted schizophrenia for both African American and white patients. Similarly, schizophrenia was negatively predicted for both races by a judgment that an organic factor was relevant. These judgments were made at equal rates for both races. There were two major differences between the patterns for African American and white patients: (1) The attribution of delusions or hallucinations without evidence of mood disturbance positively predicted schizophrenia for African American patients only; and (2) the symptom attribution of distractibility negatively predicted schizophrenia for white patients only. There were no attribution rate differences 
by race for either of these predictors, suggesting that there were judgment differences in the way they were linked to schizophrenia by clinicians for the two race groups.

Bipolar disorder results. The right side of Table 5 shows the results with the same symptom sets for the diagnosis of bipolar disorder. There were no race by symptom interactions in predicting bipolar disorder with the manic episode symptoms. Therefore, the lower right portion of Table 5 displays the overall model for both groups of patients. The model for all patients on the 13 manic episode symptoms predicting bipolar disorder was significant $\left(\mathrm{X}^{2}\right.$ $=69.276, \mathrm{df}=13, p<.001)$, correctly classifying 86 percent of the diagnoses. This result was almost entirely due to a subset of four symptoms: hypertalkativeness, psychomotor agitation, mood disorder superimposed on schizophrenia, and organic factor $\left(\mathrm{X}^{2}=\right.$ $59.524, \mathrm{df}=4, p<.001$; correctly classifying $84 \%$ of the diagnoses). In the reduced model in the lower right of Table 5, attributions of hypertalkativeness and psychomotor agitation positively predicted bipolar disorder, while judgments that the condition is superimposed on schizophrenia or the result of an organic factor decreased the odds of a bipolar diagnosis. Hypertalkativeness was attributed to white patients at a somewhat higher rate than to African American patients (marginally significant: African American $=.51$, white $=.65, \mathrm{X}^{2}$ $=3.530, \mathrm{df}=1, \mathrm{p}=.06)$. Psychomotor agitation, mood disorder superimposed on schizophrenia, and organic factor were attributed at equivalent rates to each race.

The initial model for all patients on the 19 psychotic symptoms predicting bipolar disorder was significant $\left(\mathrm{X}^{2}=50.766, \mathrm{df}=19, \mathrm{p}<\right.$ .001 ), correctly classifying 92 percent of the diagnoses. The reduced model showed that this effect was almost completely accounted for by a subset of seven symptoms: catatonic behavior, inappropriate affect, impairment of role functions, peculiar behavior, impairment of hygiene, blunted affect, and schizophrenic symptoms for more than 6 months, and organic factor $\left(\mathrm{X}^{2}=43.315\right.$, df $=7, \mathrm{p}<.001$; correctly classifying $92 \%$ of the cases). There were significant race by symptom interactions for catatonic behavior, impairment of role functions, impairment of hygiene, and blunted affect.

As for the attribution of psychotic symptoms in the diagnosis of bipolar disorder, the models were different. For African American patients, impairment of role functions and blunted affect decreased the odds of a bipolar disorder diagnosis. For white patients, inappropriate affect and peculiar behavior (which refers to actions such as talking to oneself in public) decreased the odds of a bipolar disorder diagnosis, while catatonic behavior (which refers to any unusual motor function, such as overly rigid posture or purposeless excitement) increased the odds of bipolar diagnosis. None of these attributions differed in rate between the races.

\section{DISCUSSION}

This article studied racial differences in diagnoses made by clinicians using a semistructured instrument and DSM criteria. Although we found no differences in depression, African Americans were somewhat more likely to be diagnosed with schizophrenia, while whites were much more likely than African Americans to receive a diagnosis of bipolar disorder. The multinomial logistic regression model suggests that the contrast between schizophrenia and bipolar disorder was a major contributor to this race effect. The results do not support the conclusion that race differences in diagnostic categories are completely eliminated by the use of a semi-structured diagnostic instrument. Such findings make it more difficult to attribute these patterns to misdiagnosis, although this remains a plausible explanation. Instead, these findings highlight the critical importance of clinical judgment in cross-cultural diagnosis.

This study also explored whether clinicians use symptoms differentially by patient race to construct a diagnosis. The symptom attribution analysis showed that the process clinicians used to link symptom observations to diagnostic constructs was different, particularly for schizophrenia, among African American and white patients. These differences could not be completely accounted for by race differences in symptom rates. In relating manic episode symptoms to a diagnosis of bipolar disorder, there were no race differences in symptom patterns. This scenario is consistent with the intent of the DSM to ensure that diagnoses are based on symptom evidence and the idea that semi-structured instruments reduce the variation in clinical judgment due to race. The 
major profile for this diagnosis was an individual of either race judged to be hypertalkative and agitated, with schizophrenia and organic disorder ruled out. There was, however, a slight tendency to attribute hypertalkativeness at a higher rate to white patients. This may have contributed to their higher rate of bipolar disorder.

The pattern of psychotic symptoms that was related to a diagnosis of bipolar disorder showed that somewhat different symptoms needed to be ruled out for the two patient groups. For African American patients, impairment of role functions and blunted affect were less likely to lead to a diagnosis of bipolar disorder; for white patients, inappropriate affect and peculiar behavior decreased the odds of a bipolar diagnosis. In addition, attribution of catatonic behavior (which entailed motor anomalies including agitation) increased the likelihood of a diagnosis of bipolar disorder for white but not African American patients. These findings suggest that evidence of ineffectual behavior and a flat presentation led clinicians away from a bipolar diagnosis for African Americans. For whites, an odd or inappropriate presentation led away from bipolar diagnosis. In turn, the attribution of motor anomalies increased the probability of a bipolar diagnosis only for whites.

The pattern of psychotic symptoms predicting a diagnosis of schizophrenia also differed between the two races. For African American patients, loose associations, inappropriate affect, hallucinations with voice, and vague speech increased the likelihood of a schizophrenia diagnosis. Loose associations and vague speech were positive predictors for white patients. Among these symptoms, only hallucinations with voice was attributed more frequently to African Americans. This latter finding suggests that, in distinguishing schizophrenia from other diagnoses, clinicians reported seeing auditory hallucinations in a higher proportion of their African American patients, and, in turn, this symptom was of greater importance in predicting the schizophrenia diagnosis. This is in accord with speculations in the literature that African Americans report higher rates of hallucinations, which may account for rate differences in schizophrenia by race. The finding that attribution of inappropriate affect increased the odds of a schizophrenia diagnosis for African Americans but not whites is interesting in that rates of attributing inappropriate affect did not differ between the groups. Manic episode symptoms also differed by the patient's race when used to predict schizophrenia. When African American patients were judged to exhibit delusions without mood symptoms, schizophrenia was a more likely diagnosis. For white patients, distractibility lowered the probability of a schizophrenia diagnosis.

The finding of an association between inappropriate affect and schizophrenia is noteworthy insofar as the attribution of this particular symptom was linked to the diagnosis of schizophrenia for African Americans but not for whites, even though the attribution rates did not differ by patient race. This scenario is difficult to understand if one adheres to the perspective that diagnoses are purely symptom driven. These data show that they are not. The findings suggest that the relative frequency of particular symptoms within a racial group may not be the only determinant of diagnosis (Trierweiler et al. 2000). Rather, some symptoms may be weighted differently for the two races as a matter of clinical judgment. To the extent cultural issues, such as incomplete or misunderstood communications between clinicians and patients, are the source of these weighting differences, there is the potential that diagnostic differences are a function of biases that contribute to misdiagnosis (Whaley 2002). Judgments about affect involve qualitative decisions about non-specific patient behaviors relative to a generally unspecified standard of appropriateness. Research is needed to determine if and how decisions about the quality of affect contribute to diagnostic differences for African American and white patients. Such decisions may be subject to cultural interpretation in ways clinicians need to understand better.

Symptom attribution is a complex process involving the inclusion of some observations and inferences and the exclusion of others. Our data show that both those symptoms specifically designated in the DSM as describing a disorder and those symptoms associated with an important competing diagnosis (in this case, schizophrenia versus bipolar disorder) were predictive of a diagnosis in logistic regression models. Specifically, we think that racial differences at the symptom level present unique challenges for clinicians attempting to implement a categorical system with admittedly fuzzy boundaries (American Psychiatric 
Association 1994). As a result, there is a need for more investigations that focus on the diagnostic policies employed by clinicians in order to gain deeper insights into the possible differential application of DSM criteria by patient race.

There are two important findings revealed by this study. First, a similar profile of symptoms was associated positively with a diagnosis of bipolar disorder for both racial groups. Coupled with the fact that there were no race differences in how symptoms were linked to major depression, these results suggest that the symptom-level pathway to this particular disorder is similar for both races. Any differences in a particular diagnosis must be the result of differences in the way clinicians make attributions of these symptoms. For example, hypertalkativeness was attributed more frequently to white patients, which may explain their higher rate of bipolar disorder.

Second, relatively more symptoms led toward schizophrenia and away from bipolar disorder for African Americans than whites. This pattern of results seems to indicate that efforts by clinicians to weigh important competing hypotheses may set the stage for racial biases to intercede. Interestingly, the symptoms involved in this race difference described ineffectuality (impairment of role functions), flatness (blunted affect, vague speech), and poor reality testing (hallucinations, delusion without mood, and inappropriate affect). These results are consistent with prior research showing that negative symptoms are more powerful indicators of schizophrenia for African Americans than whites (Trierweiler et al. 2000). Further research is needed to determine if such differences can be replicated in other settings where race differences in diagnosis are observed.

Finally, an interesting secondary finding to consider is that the study generated a sizable "other" diagnosis category, which included mostly suggestions of substance abuse and personality disorder. The fact that these diagnoses were not part of the study eligibility criteria suggests that, in contrast to standard hospital operating procedures, the semi-structured research instrument may have influenced clinicians to funnel patients who were not easily classifiable. This provides some evidence that semi-structured instruments may achieve reliability by rearranging decisions about cases difficult to locate within the diagnostic system. In short, the "real world" of mental health treatment involves conditions and complexities that may not be well described by instruments designed primarily to achieve satisfactory statistical properties (Trierweiler and Stricker 1998).

Taken together, the race differences found in the rate of bipolar disorder and in the pattern of symptom attribution for particular diagnoses suggest that different decision processes were at work in making diagnostic decisions for the two races. There are three possible reasons for these findings. First, African Americans are different from whites in the basic expression of symptoms. Second, selection factors operate to bring an unusual group of whites or African Americans into the urban psychiatric inpatient population. Finally, interview and information factors operate with some cases to bias the diagnosis toward schizophrenia and away from mood disorders for African American. Our ability to address the first possibility was beyond the scope of the study. Lacking a plausible theoretical rationale for the second possibility, our experience suggests that the issue of clinician bias is the most promising direction to pursue.

\section{Clinician Bias}

Some have argued that the differential treatment of African American and white patients by clinicians is an unconscious process (e.g., Schulman et al. 1999). Yet this argument is difficult to reconcile given the level of specialized training and high degree of professionalism found in most clinical settings. It is even more difficult to accept when one considers the level of attention placed on race in the United States generally and in our institutions of higher learning. The importance of racial issues has been continually emphasized at professional meetings, in special issues published by high-profile journals, in numerous books and reports, and by the organizations representing the clinical professions. It hard to imagine a board-certified clinician making a decision about an African American patient (or a patient of any other ethnic group) with no conscious consideration of how the person's race (or gender, age or socioeconomic position) might influence the clinical encounter. In fact, one experimental study found partial evidence that clinicians consciously attempted to 
overcome the influence of racial stereotyping once a hypothetical clinical case was identified as black (Abreu 1999). Similarly, a recent experiment where video-taped symptom presentations were carefully matched across patient race found that psychiatrists did not under-diagnose depression among African Americans (Kales et al. 2002). Even the most basic reading of DSM reveals information on how race and culture might be related to the diagnostic process generally as well as to specific disorders (American Psychiatric Association 1994). As a result, we think it is unlikely that clinicians are unconscious in what they do with respect to diagnosing African Americans. Rather, we think that many are not trained to do enough in this area. It may be more reasonable to assume that some clinicians might not be as critical as they should be in the realm of cultural differences. They may not be as careful, perhaps due to time constraints, in raising cultural alternatives while arriving at an initial diagnostic hypothesis. Without employing such "cultural checkpoints," some clinicians may arrive at a premature diagnostic closure due to a lack of training in how to filter what they are seeing clinically through the cultural schemas required by DSM-IV's Cultural Formulation.

Clinician bias may also stem from the tendency for clinicians to selectively attend to clinical information that is confirmatory of premature clinical hypotheses (Nurius and Gibson 1990). The application of diagnostic criteria can be influenced by clinical training and contribute to inaccuracies in the identification of pathology (Parloff, Kelman and Frank 1954; Spitzer et al. 1994; Stahler and Rappaport 1986). Differential diagnosis may also stem from data that demonstrate that African American depressed patients are more likely to present with somatic complaints while white are more likely to present with depressed mood and suicidal tendencies (Adebimpe 1981; Liss et al. 1973; Simon et al. 1973; Vitols, Waters and Keeler 1963). These studies have also indicated that hallucinations and delusions are more frequent among African Americans, which may lead to the overdiagnosis of schizophrenia for this group. Cultural suspiciousness can be an adaptive response to racism and may be misinterpreted as a sign of paranoia and lead to more frequent diagnosis of paranoid schizophrenia among African Americans compared to whites (Adebimpe
1981). Clinicians unaware of cultural variations in paranoia may view mistrust as evidence of psychosis instead of depression (Whaley 2002; Whaley 1997). Studies indicate that African Americans show less affect or more flat affect than whites, which may also increase the likelihood of receiving a diagnosis of schizophrenia (Trierweiler, et al. 2000; Welner, Liss and Robins 1973; Welner et al. 1972). Clinicians may see something that resembles "blunted affect" but may not assess the cultural accuracy of that perception by considering possible differences in the ways that African Americans and whites display emotions. What looks like "blunted affect" in an African American may be nothing more than adaptive guardedness resulting from an understandable reluctance to reveal personal information to whites.

The differentiation of bipolar disorder and schizophrenia has been identified as a particularly difficult problem (Blacker and Tsuang 1992; Fein and McGrath 1990; Pope and Lipinski 1978; Strakowski et al. 1995; Strakowski et al. 1996; Strakowski, Shelton and Kolbrener 1993). Symptoms of both bipolar disorder and schizophrenia can present in the same episode, and such comorbidity adds to the complexity of making an accurate diagnosis (Chen, Swann and Johnson 1998). Bell and Mehta (1980) were among the first to argue that clinicians misconstrue positive symptoms (e.g., persecutory delusions, auditory hallucinations, fragmented speech, pressured speech) as occurring only in schizophrenia when in fact they are also found in acutely ill bipolar patients. Jones (1986) wrote that the same symptoms may be expressed through a variety of actions determined by culture and that making a distinction between mood disturbance and schizophrenia is difficult for clinicians inexperienced with African Americans. Mukherjee et al (1983) argued that untreated mania progresses to a severely psychotic state with delusions, hallucinations, and thought disorder, making it easily confused with schizophrenia.

\section{The Importance of Clinical Judgment}

As clinicians focus primarily on symptom descriptions within DSM, they may inadequately register subtleties of communication and cultural context. Thus, empirical inaccuracies may affect decisions as judgment moves 
from observations of patient behavior and patient self-reports to symptom attributions. Our data suggest that subtle judgments about affect are of particular concern, both in terms of accuracy in reading affective states and in judging its appropriateness. Additionally, distinguishing hallucinations that indicate poor reality testing from culturally governed interpretations of subjective experience may be difficult. This would be an area where clinicians of both races are needed who are knowledgeable of ethnic, sub-cultural, and life experience situations that may confirm or modify an attribution of a symptom that may lead to a diagnosis of schizophrenia. We should expect the symptoms co-occurring with a diagnosis to be roughly comparable across groups unless there is explicit cultural reasons to believe otherwise. Work by clinicians and researchers to clarify cultural differences in symptom presentation may allow for tightening of the diagnostic system with respect to cross-cultural issues by improving symptom descriptions as applied to different populations.

The cultural issues surrounding diagnosis originate in the interpersonal interaction between clinician and patient (Rogler 1993). Naturalistic studies are needed to enhance our understanding of this interaction. Methods combining qualitative information with quantitative data are particularly promising. If race is a moderator for clinical interactions with potentially deleterious consequences for African Americans such as misdiagnosis, then work must be done to identify and describe relevant variables so that culturally tailored clinical techniques can be developed. Research on the doctor-patient interaction and interpersonal influences on self-report supports the research direction proposed here (Roter 2000; Roter and Frankel 1992; Schwartz 1999). Because race sets a context for both clinician and patient judgments about how therapeutic interactions proceed, we need to know as much as possible about how clinicians and patients of various backgrounds interpret observations and self-reports about distress, disability, and dysfunction.

Clinical judgment and misdiangosis, while related, are not quite the same thing. All diagnosis is based on expert interpretations of DSM guidelines. The term misdiagnosis implies the ability to identify unequivocally an error in judgment. However, in cases of diagnostic disagreement, it is not clear which diag- nosis is correct. By highlighting the clinical judgment process and downplaying the concept of misdiagnosis, we make the point that rather than be overly concerned with simple dichotomies of "right" and "wrong," research on race and diagnosis must focus on how to improve the clinical judgment process. Foregrounding clinical judgment while downplaying misdiagnosis places attention more appropriately on the notion that most clinicians are currently doing their best to formulate a reasonable diagnostic hypothesis within the limits of the cultural knowledge available to them. Increased emphasis on how to produce diagnoses that are more culturally informed, and thus more "credible," is more important than inferences about misdiagnosis made on the basis of anecdotal accounts or secondary data compiled from studies not designed to address issues of race. Over use of the term misdiagnosis also places too much emphasis on outcomes when in fact, skepticism about diagnosis stems more from suspicion of the process used by clinicians to make diagnoses within the context of race. Presently, we are unable to make definitive conclusions about diagnostic correctness. We must acknowledge the fact that two (or more) well-trained, culturally informed clinicians can come to reasonable, yet different, diagnostic impressions (Strakowski et al. 2002; Whaley 2002). At best, we can inspect specific instances of diagnostic difference and discuss where to assign more or less diagnostic credibility on the basis of what constitutes acceptable clinical practice.

\section{Race and the Clinical Interaction}

The mental health field has taken the position that for clinicians to become culturally competent diagnosticians, they must acknowledge differences among patients due explicitly to racial group membership (American Psychiatric Association 1994; Guarnaccia and Rodriguez 1996; Sue 1998; U.S. Department of Health and Human Services 2001). This means knowing how to word questions in terms of local idioms of distress and how to adjust the assessment of patient self-reports on the basis of things such as racial/ethnic identity, religiosity, or feelings of paranoia (Grier and Cobbs 1968; Whaley 1997). We must be very clear that in the realm of cultural variation, a "color blind" clinician will not be able 
to see clearly with respect to how to treat the racially different patient. A doctor who chooses to treat all patients the same regardless of race will also fall prey to diagnostic mistakes to the extent that any particular patient's presentation differs from the prototypical descriptions upon which current conceptualizations of mental disorder are based. In short, the field requires that clinicians be prepared to make adjustments in how they come to a diagnosis (i.e., the questions they ask, the words they use, the type and amount of information they seek, etc.) on the basis of the patient's race. This does not mean "profiling" individual African Americans by assuming, a priori, that each African American differs from the white client. Given the pervasiveness of acculturation processes in the United States, we know that not all blacks (or Latinos or whites) are alike, nor that they will always differ from the prototypical white clinical case description. The point is that clinicians must be able to make such difficult and challenging withingroup and between-group distinctions (Castillo 1997; Othmer and Othmer 1994; U.S. Department of Health and Human Services 2001). These are issues best dealt with in clinical training programs that show clinicians how to complete their tasks in culturally diverse situations (Lopez et al. 1989).

\section{Conclusions}

These issues lead one to speculate about the impact of the race of the clinician on diagnosis. Unfortunately, few empirical studies employing the design used in the present investigation have been able to recruit a racially diverse interviewing staff. As a result, the impact of race-matching between clinician and patient has yet to be fully explored. While the present study did include a racially diverse group of clinical interviewers, we were not able to match all African American patients with African American interviewers. We can, however, explore the impact of clinician race on diagnosis. Preliminary analyses of the present dataset exploring patient race differences in research diagnoses stratified by clinician race appears to show that the race of the clinician does not alter the relationship of patient race to diagnosis; African American patients remain significantly more likely to be diagnosed with schizophrenia and much less likely to receive a diagnosis of bipolar disorder irrespective of the race of the clinician making the diagnosis (Neighbors et al. 2002).

The findings reported here, and those of others (Sohler and Bromet 2000; Strakowski et al. 2002; Whaley 2002), are consistent with the argument that the use of semi-structured diagnostic instruments that are based on explicit DSM criteria does not uniformly eliminate racial disparities in diagnostic outcomes. Although we still think that the imposition of more structure influences clinicians to look at patients in a more comprehensive manner, the preconceived notions clinicians may have about patients based on race, gender, or socioeconomic status, remain an important influence on how patients are assessed.

The descriptive nature of DSM can be deceiving. The listing and specificity of the criteria make psychiatric classification appear to be a fairly straightforward and reliable process. Clearly it is not. Given the necessity of expert subjectivity in psychiatric diagnosis, culturally knowledgeable clinicians must be afforded the flexibility necessary to use their clinical skills. In short, psychiatric diagnosis with respect to assumed racial and ethnic differences must become more ethnographic (Castillo 1997; see also Fauman 1994 on the three phases in the education of a diagnostician). Until such procedures are adopted more widely by the clinical professions, we will not get to the root causes of those racial disparities that concern researchers and policy-makers. Therefore, despite the indictment of clinical judgment as the culprit in race and diagnosis, in order to implement effectively DSM-IV's Cultural Formulation (American Psychiatric Association, 1994), clinicians must be afforded the flexibility necessary to exceed the narrow focus on symptoms and probe for cultural contextual information. Allowing too much individual freedom in clinical procedures was criticized for allowing ethnocentric bias to contaminate the diagnostic process. Interestingly, and perhaps paradoxically, permitting clinician discretion in diagnostic decision making appears also to provide the best opportunity to solve the problem of misdiagnosis (Neighbors et al. 2000).

\section{Study Limitations}

The present study has a few limitations 
worth noting. First, the data were collected during the publication of DSM-III-R (American Psychiatric Association, 1987). Given DSM-IV's emphasis on cultural issues, its use during interviewer training may have had a different effect on the results. We recommend that these procedures be replicated with clinical interviewers knowledgeable about DSM-IV's cultural statements tied to particular disorders. DSM-IV also attempts to address clinician bias by the Cultural Formulation (Appendix I) which contains five cultural issues that should be addressed by clinicians: (1) ethnic group identification; (2) idioms of distress; (3) the psychosocial environment, stressors, social supports, and religion; (4) relationship between the client and the clinician; and (5) descriptions of culture bound syndromes.

Second, because of constraints on time and resources, it was impossible for this study to explore the entire range of diagnostic categories. Therefore, we cannot comment on how the issues reviewed here might be related to other important problems such as the anxiety disorders. Third, the attempt to understand how symptom level data are combined and whether this is done differentially by patient race is a promising start, but the results are not conclusive. In particular, the post hoc nature of the analysis of symptom-diagnosis linkages may have selected for chance differences on some of the variables. However, the overall tendency to describe African Americans in terms of schizophrenia and whites in terms of bipolar disorder appears to be a reliable result providing intriguing leads that warrant further investigation by researchers working in different settings.

Fourth, the use of third and fourth year psychiatric residents as clinical investigators may be seen as both a limitation and an asset. While our results are consistent with studies that have used more experienced clinical interviewers (Sohler and Bromet 2000; Strakowski et al. 2002), an argument could be made that these are less experienced clinicians and that the results presented here are merely artifacts of the clinicians' low levels of training. On the other hand, it is possible that, precisely because these clinicians were more recently trained, they were more likely to adhere to the guidelines of DSM-III-R and, as such, less likely than more experienced clinicians to apply their own idiosyncratic interpretations to the criteria. These competing interpretations are diffi- cult to resolve, but they do speak to the necessity of replicating the study design with different types of clinicians.

Fifth, the investigation was conducted in one clinical setting, which is not unusual for studies of this type. This shortcoming can only be addressed by investing in larger multi-site collaborative projects. This raises some question about the degree to which these results can be generalized to other treatment or research settings. The city of Detroit has its own unique history, which undoubtedly contributed to the wider socio-political context within which the mental health system operated. Such factors affect who receives treatment and where. These selection forces were clearly in operation in the Detroit urban social setting at the time these date were collected and, as a result, may have had an impact on the racial make-up the sample. In fact, these socio-political factors made it much more difficult to obtain data on whites than on African Americans (Thompson et al. 1996). Nevertheless, the results reported here are consistent with more recent work showing racial differences in diagnostic outcomes under more controlled interviewing conditions (Sohler and Bromet 2000; Strakowski et al. 2002; Whaley 2002). This suggests that our results are not overly idiosyncratic.

The fact that our results are consistent with those of other studies raises another important point. The instrument used in this study (the DSM-III-R Symptom Checklist) can be characterized as less structured than the more widely used Structured Clinical Interview for DSM-IV and the Diagnostic Interview for Genetic Studies. An argument can be made that these more rigid semi-structured diagnostic instruments would have produced a different pattern of results had they been employed in the present study. This is certainly plausible but not likely. In fact, the differences among the DSM-III-R Symptom Checklist, the Structured Clinical Interview for DSM-IV and the Diagnostic Interview for Genetic Studies are not all that large; these diagnostic tools are more similar than different in that they all allow for the use of clinical judgment. If there is a contrast to be made, it is between these semi-structured instruments designed to be used by clinicians and the much more highly structured epidemiologic instruments such as the Composite International Diagnostic Interview or the Diagnostic Interview Schedule. 
NOTE

1. We use the term "race" to refer to a socially constructed category of limited biological and genetic significance because there is as much genetic variation within any socalled "racial" group as there is between any two groups. From this perspective, "race" overlaps considerably with the concept of ethnicity. Certainly there is considerable ethnic variation within both the socalled "African American" and "white" groups but it is beyond the scope of the present investigation to present such withingroup ethnic differences at this time. We opt in this paper to employ the term race as a convenient descriptor to refer to a research variable shown to be associated with important mental health outcomes. While others have argued to eliminate the word race, this would be inappropriate for the present paper since the analyses were conducted upon respondents who were asked to selfidentify within the traditional U.S. "racial" categories and not to differentiate themselves in terms of their specific ethnic group memberships. In this study, race was operationally defined by self-identification of the respondent using the following item: "People use different words to refer to their race or ethnic group. What word or words do you use?." In response, 74 percent of the European American patients preferred the term "white," 7 percent said "European" (not European American), and none said Caucasian. Ninety-two percent of the black patients chose black/African American as their preferred term. As a result, we use the terms African American and white in this paper.

\section{REFERENCES}

Abreu, Jose M. 1999. "Conscious and Nonconscious African American Stereotypes: Impact on First Impression and Diagnostic Ratings by Therapists." Journal of Consulting and Clinical Psychology 67:387-93.

Adebimpe, Victor. 1981. "Overview: white Norms and Psychiatric Diagnosis of Black Patients." American Journal of Psychiatry 138:279-85.

—. ed. 1982. Psychiatric Symptoms in Black Patients. New York: Plenum.

Adebimpe, Victor, Helen Klein, and J. Fried. 1981. "Hallucinations and Delusions in Black
Psychiatric Patients." Journal of the National Medical Association 73:517-20.

American Psychiatric Association. 1980. Diagnostic and Statistical Manual of Mental Disorders. 3rd ed. Washington, DC: American Psychiatric Association.

American Psychiatric Association. 1987. Diagnostic and Statistical Manual of Mental Disorders. 3rd ed, rev. Washington, DC: American Psychiatric Association.

American Psychiatric Association. 1994. Diagnostic and Statistical Manual of Mental Disorders. 4th ed. Washington, DC: American Psychiatric Association.

Bell, Carl and H. Mehta. 1980. "The Misdiagnosis of Black Patients with Manic Depressive Illness." Journal of the National Medical Association 72:141-45.

1981. "Misdiagnosis of Black Patients with Manic Depressive Illness: Second in a Series." Journal of the National Medical Association 73:101-07.

Blacker, Deborah., and Ming T. Tsuang. 1992. "Contested Boundaries of Bipolar Disorder and the Limits of Categorical Diagnosis in Psychiatry." American Journal of Psychiatry 149:1473-83.

Blue, A. V., and Atwood D. Gaines. 1992. "The Ethnopsychiatric Repertoire: a Review and Overview of Ethnopsychiatric Studies." in Ethnopsychiatry: The Cultural Construction of Professional and Folk Psychiatries, edited by A.D. Gaines. Albany: State University of New York Press.

Carter, James E. 1995. "Psychosocial/Cultural Issues in Medicine and Psychiatry: Treating African Americans." Journal of the National Medical Association 87:857-60.

Carter, James H. 1974. "Recognizing Psychiatric Symptoms in Black Americans." Geriatrics 29:96-99.

Castillo, Richard J. 1997. Culture \& Mental Illness: a Client-Centered Approach. Pacific Grove, CA: Brooks/Cole Publishing Company.

Chen, Richard Y. Alan. C. Swann, and Bankole A. Johnson. 1998. "Stability of Diagnosis in Bipolar Disorder." Journal of Nervous and Mental Disease 186:17-23.

Fabrega, Horacio, Jr. 1996. "Cultural and Historical Foundations of Psychiatric Diagnosis.” Pp. 3-14 in Culture and Diagnosis: a DSM-IV Perspective, edited by J. E. Mezzich, A. Kleinman, H. Fabrega, Jr., and D. L. Parron. Washington, DC: American Psychiatric Association Press.

Fauman, Michael A. 1994. Study Guide to DSM-IV. Washington, DC: American Psychiatric Press, Inc.

Fein, Sidney and Michael G. McGrath. 1990. "Problems in Diagnosing Bipolar Disorder in Catatonic Patients." Journal of Clinical Psychiatry 51:203-05. 
Fischer, Joel. 1969. "Negroes and whites and Rates of Mental Illness: Reconsideration of a Myth." Psychiatry 32:428-46.

Gaines, Atwood D. 1992. "From DSM-I To DSMIII-R: Voices of Self, Mastery, and the Other: a Cultural Constructivist Reading of U.S. Psychiatric Classification." Social Science and Medicine 35:3-24.

Garb, Howard N. 1997. "Race Bias, Social Class Bias, and Gender Bias in Clinical Judgment." Clinical Psychology: Science and Practice 4:99-120.

Grier, William H. and Price M. Cobbs. 1968. Black Rage. New York: Basic Books.

Griffith, Ezra E. H. 1996. "African American Perspectives." Pp. 27-29 in Culture and Psychiatric Diagnosis: a DSM-IV Perspective, edited by J. E. Mezzich, A. Kleinman, H. Fabrega, Jr., and D. L. Parron. Washington, D.C.: American Psychiatric Association Press.

Guarnaccia, Peter J. and Orlando Rodriguez. 1996. "Concepts of Culture and Their Role in the Development of Culturally Competent Mental Health Services." Hispanic Journal of Behavioral Sciences 18:419-43.

Heider, Fritz 1958. The Psychology of Interpersonal Relations. New York: John Wiley \& Sons.

Hosmer, David W., and Stanley Lemeshow. 1989. Applied Logistic Regression. New York: Wiley.

Hudziak, James J., John E. Helzer, Martin W. Wetzel, Keith B. Kessel. et al. 1993. "The Use of the DSM-III-R Checklist for Initial Diagnostic Assessments." Comprehensive Psychiatry 34:375-83.

Jones, Billy and Beverly Gray. 1986. "Problems in Diagnosing Schizophrenia and Affective Disorders Among Blacks." Hospital and Community Psychiatry 37:61-5.

Kales, Helen C., Harold W. Neighbors, F. C. Blow, K. Khanuja, L. Gillon, D. E. Welsh, C. R. Bingham, S. H. Maixner, and A. Mellow. 2002. "The Effect of Race and Gender on Psychiatrists' Diagnosis and Treatment of Major Depression in the Elderly." Ann Arbor: Serious Mental Illness Research Education and Clinical Center, Health Services Research and Development, VA Healthcare System; Division of Geriatric Psychiatry.

Kirk, Stuart A. and Herb Kutchins. 1992. The Selling of DSM: The Rhetoric of Science in Psychiatry. New York: Aldine de Guyter.

Kleinman, Arthur 1996. "How Is Culture Important For DSM-IV?" Pp. 15-25 in Culture and Diagnosis: a DSM-IV Perspective, edited by J.E. Mezzich, A. Kleinman, H. Fabrega, Jr., and D. Parron. Washington, DC: American Psychiatric Association Press.

Kramer, M., B. Rosen, and E. Willis. 1973. Definitions and Distributions of Mental Disorders in a Racist Society. Pittsburgh: University of Pittsburgh Press.
Lawson, William 1986. "Racial and Ethnic Factors in Psychiatric Research." Culture, Medicine, and Psychiatry 37:50-4.

Lipton, Alan A. and Franklin S. Simon. 1985. "Psychiatric Diagnosis in a State Hospital: Manhattan State Revisited." Hospital and Community Psychiatry 36:368-73.

Liss, Jay, Amos Welner, Eli Robins, and Marsha Richardson. 1973. "Psychiatric Symptoms in white and Black Inpatients I: Record Study." Comprehensive Psychiatry 14:475-81.

Lock, Margaret 1987. "DSM-III as a Culture-Bound Construct: Commentary on Culture-Bound Syndromes and International Disease Classifications." Culture, Medicine and Psychiatry 11:35-42.

Lopez, Steven R., K. Pany Grover, Debra Holland, Melissa J. Johnson, C. D. Kan, K. Kanel, C. A. Mellins, and M. C. Rhyne. 1989. "Development of Culturally Sensitive Psychotherapists." Professional Psychology: Research and Practice 20:369-76.

Loring, Marti and Brian Powell. 1988. "Gender, Race, and DSM-III: a Study of Objectivity of Psychiatric Diagnostic Behavior." Journal of Health and Social Behavior 29:1-22.

Lukoff, David, Francis Lu, and Robert Turner. 1992. "Toward a More Culturally Sensitive DSM-IV: Psychoreligious and Psychospiritual Problems." Journal of Nervous and Mental Disease 180:673-82.

Mezzich, Juan E., Arthur Kleinman, Horacio Fabrega, Jr., and Delores L. Parron, eds. 1996. Culture and Psychiatric Diagnosis: a DSM-IV Perspective. Washington, DC: American Psychiatric Association Press.

Mukherjee, Sukdeb, Sashi Shukla, J. Woodle, Aarnold M. Rosen, and Silvia Olarte. 1983. "Misdiagnosis of Schizophrenia in Bipolar Patients: a Multiethnic Comparison." American Journal of Psychiatry 140:1571-74.

Neighbors, Harold W., Briggett Ford, Steven J. Trierweiler, and Phyllis Stillman. 2002. "African American Mental Health Disparities: Prevalence, Utilization, Diagnosis \& Treatment." in Eliminating Disparities in the Treatment of African Americans with Mental Health and Substance Abuse Disorders, edited by J. Berry, D. Baldwin, and P. Marshall. Rockville, MD: Center for Mental Health Services. Substance Abuse \& Mental Health Services Administration.

Neighbors, Harold W., James S. Jackson, Linn Campbell, and Donald Williams. 1989. "Racial Influences On Psychiatric Diagnosis: a Review and Suggestions For Research." Community Mental Health Journal 25:301-11.

Neighbors, Harold W., Steven J. Trierweiler, Cheryl Munday, Briggett Ford, Phillip J. Bowman, Victoria J. Binion, and Phyllis K. Stillman. 2000. "Psychopathology and the Diagnosis of African 
Americans: DSM Criteria, Clinical Judgment, and Cultural Context." Ann Arbor MI: Institute for Social Research, University of Michigan.

Neighbors, Harold W., Steven J. Trierweiler, Cheryl Munday, Estina E. Thompson, James S. Jackson, Victoria J. Binion, and John Gomez. 1999. "Psychiatric Diagnosis of African Americans: Diagnostic Divergence in Clinician Structured and Semistructured Interviewing Conditions." Journal of the National Medical Association 91:601-12.

Nurius, Paula S. and John W. Gibson. 1990. "Clinical Observation, Inference, Reasoning, and Judgment in Social Work: An Update." Social Work Research \& Abstracts 26:18-25.

Othmer, Ekkehard and Sieglinde C. Othmer. 1994. The Clinical Interview: Using DSM-IV, Volume 1Fundamentals. Washington, DC: American Psychiatric Association Press.

Parloff, Morris B., Herbert C. Kelman, and Jerome D. Frank. 1954. "Comfort, Effectiveness, and Self-Awareness As Criteria of Improvement in Psychotherapy." American Jounral of Psychiatry 111:343-52.

Pope, Harrison G. and Joseph F. Lipinski. 1978. "Diagnosis of Schizophrenia and Manic Depressive Illness." Archives of General Psychiatry 35:811-27.

Raskin, Allen., Thomas H. Crook, and Kenneth D. Herman. 1975. "Psychiatric History and Symptom Differences in Black and white Depressed Inpatients." Journal of Consulting and Clinical Psychology 43:73-80.

Rogler, Lloyd H. 1993. "Culturally Sensitizing Psychiatric Diagnosis: a Framework For Research." Journal of Nervous and Mental Disease 181:401-08.

Roter, Debra 2000. "The Enduring and Evolving Nature of the Patient-Physician Relationship." Patient Education and Counseling 39:5-15.

Roter, Debra and Richard Frankel. 1992. "Quantitative and Qualitative Approaches to the Evaluation of Medical Dialogue." Social Science and Medicine 34:1097-1103.

Schulman, Kevin A., Jesse A. Berlin, William Harless, Jon F. Kerner, Shyrl Sistrunk, Bernard J. Gersh, Ross Dube, Christopher K. Taleghani, Jennifer E. Burke, Sankey Williams, John M. Eisenberg, and Jose J. Escarce. 1999. "The Effect of Race and Sex On Physicians' Recommendations for Cardia Catheterization." The New England Journal of Medicine 340:618-25.

Schwartz, Norbert 1999. "Self-Reports: How the Questions Shape the Answers." American Psychologist 54:93-105.

Simon, Robert J., Joseph L. Fleiss, Barry J. Gurland, Pamela R. Stiller, and Lawrence Sharpe. 1973. "Depression and Schizophrenia in Hospitalized Black and White Mental Patients." Archives of General Psychiatry 28:509-12.
Smedley, Brian D., Adrienne Y. Stith, and Alan R. Nelson. 2002. "Unequal Treatment: Confronting Racial and Ethnic Disparities in Health Care (Summary)." Pp. 1-28. Washington, DC: Institute of Medicine, National Academy Press.

Snowden, Lonnie R. and Freda K. Cheung. 1990. "Use of Inpatient Mental Health Services by Members of Ethnic Minority Groups." American Psychologist 45:347-55.

Sohler, Nancy L. and Evelyn J. Bromet. 2000. "Does Racial Bias Influence Clinical Diagnoses Assigned to Psychiatric Inpatients?" Annual Meeting of the American Public Health Association. Boston, MA.

Spitzer, Robert L. Miriam Gibbon, Andrew E. Skodol, Janet B. Williams, and Michael B. First. 1994. DSM-IV Casebook: a Learning Companion to the DSM Fourth Edition. Washington, DC: American Psychiatric Association Press.

Stahler, Gerald J. and Herbert Rappaport. 1986. "Do Therapists Bias Their Ratings of Patient Functioning Under Peer Review?" Community Mental Health Journal 22:265-74.

Strakowski, Stephen M., Paul E. Keck, Lesley M. Arnold, J. Collins, Daniel R. Wilson, David E. Fleck, K. B. Corey, J. Amicone, and Victor R. Adebimpe. 2003. "Ethnicity and Diagnosis in Patients With Affective Psychoses." Journal of Clinical Psychiatry 64:747-54.

Strakowski, Stephen M., Heather Lonczak, Kenji Sax, Scott A. West, A. Crist, R. Mehta, and O. Thienhaus. 1995. "The Effects of Race on Diagnosis and Disposition from a Psychiatric Emergency Service." Journal of Clinical Psychiatry 56:101-07.

Strakowski, Stephen M., Susan L. McElroy, Paul E. Keck, and Scott A. West. 1996. "Racial Influence on Diagnosis in Psychotic Mania." Journal of Affective Disorders 39:157-62.

Strakowski, Stephen M., Richard C. Shelton, and Meridith L. Kolbrener. 1993. "The Effects of Race and Comorbidity on Clinical Diagnosis in Patients With Psychosis." Journal of Clinical Psychiatry 54:96-102.

Sue, Stanley. 1998. "In Search of Cultural Competence in Psychotherapy and Counseling." American Psychologist 53:440-48.

Sue, Stanley, and Nolan Zane. 1987. "The Role of Culture and Cultural Techniques in Psychotherapy: a Critique and Reformulation." American Psychologist 42:37-45.

Sussman, Linda K., Lee N. Robins, and Felton Earls. 1987. "Treatment-seeking for Depression by Black and White Americans." Social Science and Medicine 24:187-96.

Thomas, Alexander and Samuel Sillen. 1972. Racism and Psychiatry. New York: Bruner/Mazel.

Thompson, Estina E., Harold W. Neighbors, Cheryl Munday, and James S. Jackson. 1996. 
"Recruitment and Retention of African American Patients for Clinical Research: An Exploration of Response Rates in an Urban Psychiatric Hospital.” Journal of Consulting and Clinical Psychology 64:861-67.

Tonks, Clive M., Eugene S. Paykel, and Gerald L. Klerman. 1970. "Clinical Depression Among Negroes." American Journal of Psychiatry 127:329-35.

Trierweiler, Steven J., Harold W. Neighbors, Cheryl Munday, Estina E. Thompson, Victoria J. Binion, and John P. Gomez. 2000. "Clinician Attributions Associated with Diagnosis of Schizophrenia in African American and NonAfrican American Patients." Journal of Consulting and Clinical Psychology 68:171-75.

Trierweiler, Steven J. and George Stricker. 1998. The Scientific Practice of Professional Psychology. New York: Plenum.

Tseng, Wen-Shing. 1996. "Cultural Comments On Mood and Anxiety Disorders-I.” Pp. 115-121 in Culture and Diagnosis: a DSM-IV Perspective, edited by J.E. Mezzich, A. Kleinman, H. Fabrega, Jr., and D. Parron. Washington, DC: American Psychiatric Association Press.

U.S. Department of Health and Human Services. 2001. "Mental Health: Culture, Race, and Ethnicity - a Supplement to Mental Health: a Report of the Surgeon General." Rockville, MD: U.S. Department of Health and Human Services, Substance Abuse and Mental Health Services
Administration, Center for Mental Health Services.

van Ryn, Michelle and Jane Burke. 2000. "The Effect of Patient Race and Socio-economic Status on Physician's Perceptions of Patients." Social Science and Medicine 50:813-28.

Vitols, M. M., H. G. Waters, and M. H. Keeler. 1963. "Hallucinations and Delusions in white and Negro Schizophrenics." American Journal of Psychiatry 120:472-76.

Welner, Amos, Jay L. Liss, and Eli Robins. 1973. "Psychiatric Symptoms in White and Black Inpatients II: Follow-up Study." Comprehensive Psychiatry 14:483-88.

Welner, Amos, Jay L. Liss, Eli Robins, and Marsha Richardson. 1972. "Undiagnosed Psychiatric Patients Part I: Record Study." British Journal of Psychiatry 120:315-19.

Whaley, Arthur L. 1997. "Ethnicity/Race, Paranoia, and Psychiatric Diagnosis: Clinician Bias Versus Sociocultural Differences." Journal of Psychopathology and Behavioral Assessment 19:1-20.

- 2002. "The Culturally-sensitive Diagnostic Interview Research Project: A Study on the Psychiatric Misdiagnosis of African American Patients." African American Research Perspectives 8:57-66.

Worthington, Cassandra. 1992. "An Examination of Factors Influencing the Diagnosis and Treatment of Black Patients in the Mental Health System." Archives of Psychiatric Nursing 6:195-204.

Harold W. Neighbors is Associate Professor of Health Behavior and Health Education in the School of Public Health at the University of Michigan. He is Associate Director for Research Training with the Center for Research on Ethnicity, Culture, and Health in the School for Public Health, where he is Principal Investigator of two NIH training programs, Promoting Ethnic Diversity in Public Health Training and the Michigan Bridge to the Doctorate Program. Dr. Neighbors directs the School's Paul B. Cornely Postdoctoral Program. Dr. Neighbors is also Associate Director of the Program for Research on Black Americans at the Institute for Social Research. His research interests and areas of publication include psychiatric epidemiology with an emphasis on ethnic and cultural influences on the assessment (diagnosis and case finding) of mental disorder and the utilization of informal and professional services by African Americans. He is coeditor (with James S. Jackson) of the book, Mental Health in Black America (1996, Sage).

Steven J. Trierweiler is Associate Research Scientist in the Institute for Social Research at the University of Michigan. He also is a licensed clinical psychologist. His research focuses on clinical judgment, culture, and communication issues in the diagnosis and treatment of African Americans. He is principal author (with George Stricker) of the book, The Scientific Practice of Professional Psychology (1998, Plenum).

Briggett C. Ford is Assistant Professor in the School of Social Work at the University of Michigan-Ann Arbor. Her research interests include the epidemiology of mental disorders, disparities in mental health, posttraumatic stress disorder and the mental health consequences of exposure to violence for African American women.

Jordana R. Muroff is a doctoral student in social work and psychology at the University of Michigan. She is a pre-doctoral fellow in the Race and Mental Health NIMH Training Program. She is currently a post master's fellow in the Child and Adolescent Psychiatry Outpatient Division and Adult Anxiety Disorders Programs of the University of Michigan Hospital. 